\title{
Pós-graduação em ciência do solo na região amazônica: uma possivel realidade
}

\section{Graduate study in soil science in the Amazon region: a possible reality}

\section{Postgrado en ciencia del suelo en la región amazónica: una realidad posible.}

Franciane Diniz Cogo, doutoranda do Programa de PósGraduação em Ciência do Solo da Universidade Federal de Lavras / Departamento de Ciência do Solo. Endereço: Universidade Federal de Lavras/Departamento de Ciência do Solo, Caixa Postal 3037. CEP: 37200-000 - Lavras, MG. Telefone: (35) 2142-2051. E-mail: fdcogo@yahoo.com.br.

Helder Barbosa Paulino, doutor em Fitotecnia, professor associado I na Universidade Federal de Goiás / Campus Jataí. Endereço: UFG/ Campus Jataí, Distrito Industrial. CEP: 75200-000 - Jataí, GO. Telefone: (64) 3606-8202. E-mail: helderlino51@yahoo.com.br.

Marco Aurélio Carbone Carneiro, doutor em Ciência do Solo pela Universidade Federal de Lavras, professor associado III na UFLA/ DCS. Endereço: Universidade Federal de Lavras/Departamento de Ciência do Solo, Caixa Postal 3037. CEP: 37200-000 - Lavras, MG. Telefone:(35) 2142-2051. E-mail: marcocarbone@dcs.ufla.br.

\section{Resumo}

O presente estudo objetivou verificar a existência de programas de pós-graduação na temática Ciência do Solo e/ou cursos correlatos, bem como o perfil de docentes dos programas na região amazônica do Brasil, de modo a auxiliar futuras ações para o desenvolvimento da pesquisa em Ciência do Solo. Nessa região, encontraram-se 13 
programas de pós-graduação, com 39 disciplinas oferecidas que apresentam aderência ao tema Ciência do Solo, lecionadas por 36 docentes, indicando que há professores qualificados para a abertura de programa de pós-graduação em Ciência do Solo. Para a concretização de tal objetivo, políticas de incentivo e financiamento para o fortalecimento dos grupos de pesquisa com foco no desenvolvimento da Ciência do Solo regional contribuirão para a redução das assimetrias, geração de pesquisas de importância regional, formação de recursos humanos de elevada qualidade e fixação de doutores na região.

Palavras-chave: Ensino de Ciência do Solo. Programa de Pós-Graduação. Docentes.

\section{Abstract}

The present study aimed to verify the existence of graduate programs dealing with the theme of Soil Science and related fields, as well as the profile of professors in these programs in the Amazon region of Brazil, in order to facilitate the future development of research in Soil Science. In this region 13 Graduate Programs were found, with 39 subjects offered adhering to the theme of Soil Science, taught by 36 professors, thereby indicating that there are qualified professors for the opening of a Graduate Program in Soil Science. To achieve this objective, policies of incentives and funding designed to strengthen research groups which focus on the development of regional Soil Science will contribute to the reduction of disparities, the generation of research of regional importance, the training of human resources of high quality, and the fixation of doctors in the region.

Keywords: Education of Soil Science. Postgraduate Program.Professors.

\section{Resumen}

El presente estudio tuvo como objetivo verificar la existencia de programas de posgrado en Ciencia del Suelo y / o cursos relacionados, así 
como el perfil de los docentes de los programas en la región amazónica de Brasil, con el fin de ayudar a las futuras acciones para el desarrollo de la investigación en Ciencia del Suelo. En esta región se encuentran 13 programas de posgrado, con 39 asignaturas que están relacionadas con la temática de la Ciencia del Suelo, impartidas por 36 docentes, lo que indica que hay maestros calificados para la apertura del Programa de Posgrado en Ciencia del Suelo. Para lograr tal objetivo, políticas de incentivos y fondos para fortalecer los grupos de investigación que se centran en el desarrollo de la Ciencia del Suelo podrán contribuir para reducir las disparidades, la generación de investigación de importancia regional, la formación de recursos humanos de alta calidad y la retención de los médicos en la región.

Palabras clave: Enseñanza de Ciencias de Suelo. Programa de Posgrado. Docentes.

\section{Introdução}

O Plano Nacional de Pós-Graduação 2011-2020 do Brasil, instrumento que norteia as políticas públicas nacionais relacionadas à pós-graduação, possui como finalidade a expansão do Sistema Nacional de Pós-Graduação, a primazia da qualidade, a quebra da endogenia e a atenção à redução das assimetrias. No Brasil, a pós-graduação compreende 4.700 cursos autorizados pelo MEC/Capes, entre mestrados, doutorados acadêmicos e mestrados profissionais - abrangendo 79 áreas de conhecimento. Apesar desses números, somente 5\% do total está localizado na Amazônia. Esse fato demonstra a necessidade de avaliação dos motivos associados às políticas para o incremento da Pós-Graduação em Ciência do Solo nessa importante região para o País, para se desenvolver pesquisas sobre a temática no âmbito local, como tem havido em outras regiões com programas consolidados (MORAES; GIROLDO, 2012).

A região amazônica do Brasil, que compreende os estados do Amazonas, Acre, Rondônia, Roraima, Amapá, Pará, o extremo sul do Tocantins, praticamente todo o Mato Grosso e o oeste do Maranhão, em 
uma área de aproximadamente 5,1 milhões de quilômetros quadrados (cerca de 60\% do território nacional), é considerada detentora da maior biodiversidade do mundo (MITTERMEIER et al., 1992). No entanto, possui o menor índice de desenvolvimento econômico do País. A respeito disso, comenta a FAO em relatório de 2013: "a desigualdade de rendimentos, educação, saúde e outros indicadores persiste de uma geração à outra e se apresenta num contexto de baixa mobilidade socioeconômica”. Assim, com o intuito de reduzir tais desigualdades, políticas que incentivem a criação e a consolidação da formação de recursos humanos na região tornam-se necessárias para o desenvolvimento científico e tecnológico local.

Nessa região encontram-se 232 programas de pós-graduação, sendo 51 em nível de doutorado, 173 em nível de mestrado e oito em nível de mestrado profissionalizante. Embora representem pequena fração dos programas da pós-graduação brasileira, os cursos de educação superior continuada cresceram na região cerca de $30 \%$, enquanto que a média nacional foi de 20,8\%. Nos últimos sete anos, notou-se um salto no número de programas de pós-graduação na Amazônia de 92 para 232, o que significa um aumento de mais de 150\%, embora não haja programas voltados especificamente à área de Ciência do Solo.

Analisando apenas os cursos de doutorado, o crescimento é ainda mais relevante: cerca de $200 \%$, graças à ampliação do número de cursos (eram 17 em 2003 e passaram para 51 em 2010). Apesar desse aumento, ainda não são contempladas 23 (30\%) das 79 áreas de conhecimentos consideradas pela Capes, entre as quais Ciência do Solo, área vital para o desenvolvimento sustentável regional, uma vez que em parte dessa região há o avanço da fronteira agrícola pela pressão do agronegócio, e mesmo para o melhor entendimento dos processos de conservação da própria biodiversidade que ocorre no solo.

Em 2014, a Pós-Graduação em Ciência do Solo no Brasil completará 50 anos, abrangendo 16 programas (CAPES, 2009), sendo 13 com cursos de mestrado e doutorado e três apenas com mestrado, concentrados nas regiões Sul (25\%), Sudeste (31\%) e Nordeste (43\%) (CARNEIRO; SOUZA; PAULINO, 2011). Desses programas de pós- 
graduação em Ciência do Solo, 50\% foram bem avaliados, recebendo conceitos 5, 6 e 7, o que indica a eficiência e a qualidade das instituições de ensino, dos docentes e dos discentes. Entretanto, ainda há um longo caminho a ser percorrido, afinal, o País tem um número reduzido de programas de pós-graduação com esses conceitos, o que deve ser ampliado caso se busque excelência nas pesquisas científicas com foco no uso sustentável do solo, nas diferentes regiões brasileiras.

A contribuição da Ciência do Solo para o desenvolvimento nos âmbitos agrícola e ambiental é notável, uma vez que visa estreitar o equilíbrio da exploração racional das diferentes classes de solo, principalmente quando se trata do desenvolvimento do agronegócio. Assim, a pesquisa na área de Ciência do Solo interfere de maneira direta na segurança alimentar, além de garantir, com métodos e avaliações realizadas no País, o uso sustentável do solo, graças ao desenvolvimento de novas tecnologias de uso e manejo de solo. Nesse sentido, grandes avanços foram realizados nos setores agrícolas por meio da contribuição das áreas da Ciência do Solo, que permitiram o melhor manejo e uso das diferentes classes de solos tropicais, a utilização eficiente dos fertilizantes e o aumento de produção de grãos, carne, fibras e biocombustiveis, proporcionando incremento na competitividade brasileira no comércio mundial de alimentos (FAO, 2012).

A importância dos programas de pós-graduação em Ciência do Solo está demonstrada em suas realizações nas regiões Sul, Sudeste e Nordeste e na contribuição significativa para o desenvolvimento agrícola e econômico dessas localidades, promovendo direta e indiretamente um uso produtivo e racional das principais classes de solos, sempre com foco em sistemas conservacionistas, na administração racional dos fertilizantes e nos meios sustentáveis de produção.

O presente estudo objetivou verificar a existência e o perfil dos programas de pós-graduação em Ciência do Solo e/ ou cursos correlatos, bem como o perfil dos docentes desses programas na região amazônica, de modo a auxiliar ações futuras para o desenvolvimento da pesquisa em Ciência do Solo nessa região do Brasil. 


\section{Material e métodos}

O presente estudo caracteriza-se como documento exploratório e descritivo, visa identificar os Programas de Pós-Graduação (PPG) em Ciência do Solo, stricto sensu, e/ou programas de pós-graduação que apresentam disciplinas relacionadas a essa área de conhecimento localizados na região amazônica.

A pesquisa apresentou duas etapas, sendo que a primeira consistiu no levantamento e na identificação dos PPGs de mestrado e/ ou doutorado em Ciência do Solo e de outros cursos de pós-graduação com disciplinas relacionadas à Ciência do Solo. Foram tomados como base relatórios enviados à Capes no triênio 2007-2010 e disponibilizados no site http:/ / www.capes.gov.br/cursos-recomendados, no período compreendido entre dezembro 2012 e janeiro de 2013. Para a identificação dos programas de pós-graduação com disciplinas relacionadas à Ciência do Solo, foram consultadas as páginas on-line de todos os PPGs localizados na região amazônica.

Na segunda etapa, realizou-se uma caracterização do perfil acadêmico dos docentes que lecionavam disciplinas ligadas à Ciência do Solo. Para tanto, procedeu-se aos levantamentos dos currículos dos professores dos programas de pós-graduação da região identificados na primeira etapa e disponibilizados na Plataforma Lattes, no portal do Conselho Nacional de Desenvolvimento Científico e Tecnológico (CNPq), no seguinte endereço eletrônico: www.cnpq.br.

Com a finalidade de caracterizar os docentes, foram analisados os seguintes parâmetros: formação ou titulação acadêmica (graduação, mestrado e doutorado), que, para uma melhor disposição das informações, foram organizados em planilhas, tabelas e gráficos, no Programa Microsoft Excel®.

\section{Resultados e discussão}

Dos 16 programas de pós-graduação em Ciência do Solo no Brasil reconhecidos pela Capes, nenhum se encontra na região amazônica 
(Figuras 1 e 2). Considerando a importância econômica agrícola da região e a diversidade de classes de solo, pois a Amazônia representa quase $60 \%$ do território brasileiro e contribui com $8 \%$ do PIB nacional, a ausência de programas de pós-graduação pode comprometer/dificultar o desenvolvimento sustentável local e provocar, por falta de pesquisas voltadas ao manejo sustentável dos solos, a degradação de extensas áreas, como já tem ocorrido devido à prática extrativista e ao uso inadequado dos solos.

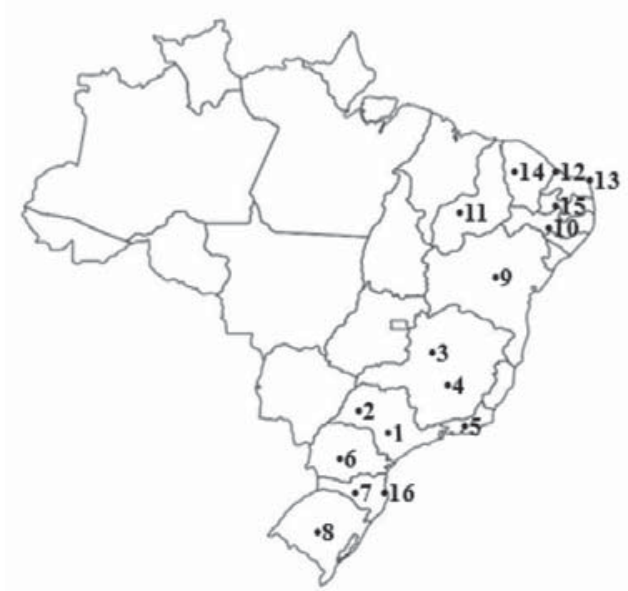

Obs.: 1: USP, 2: Unesp, 3: Ufla, 4: UFV, 5: UFRRJ, 6: UFPR, 7: Udesc, 8: UFRGS, 9: UFRB, 10: UFPE, 11: UFPI, 12 e 13: Ufersa, 14: UFC, 15: UFPB e 16: UFSM

Figura 1. Distribuição geográfica das instituições com programas em pós-graduação em Ciência do Solo no Brasil

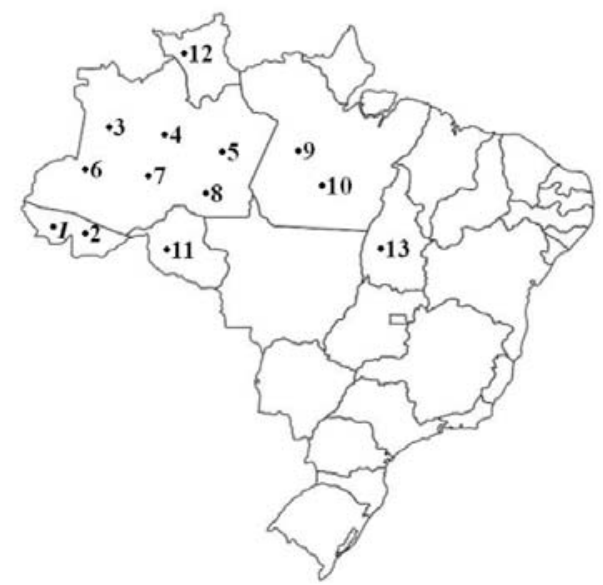

Obs.: Os números no mapa correspondem à numeração dos cursos indicados na Tabela 1.

Figura 2. Distribuição geográfica dos programas de pós-graduação com disciplinas relacionadas à Ciência do Solo. 
Além disso, a Amazônia é sede da maior extensão de florestas tropicais do mundo (LAURANCE et al, 2001) e abriga uma grande proporção da biodiversidade mundial. Apesar de sofrer com desmatamento de forma substancial nas últimas décadas (ACHARD et al., 2002) e também de estar sendo incorporada a sistemas produtivos, não há um correspondente estudo do uso e manejo sustentável do solo e da sustentabilidade para esse bioma. Tal fato pode comprometer ainda mais essas áreas, promovendo, a curto e médio prazo, a degradação de solos em extensas áreas, caso o manejo seja inadequado. Isso corrobora os estudos sobre a aptidão agrícola de solos do Brasil, que mostram que 70\% da degradação do solo refere-se à perda da camada superficial por processos erosivos, devido ao manejo inadequado e ao uso de solo sem aptidão agrícola (MANZATTO; FREITAS JUNIOR; PERES, 2002). Assim, o futuro da biodiversidade na Amazônia brasileira está atualmente em uma crítica de conjuntura (OLIVER; DANIEL; ROBERT, 2012).

Nota-se ainda que a pesquisa em Ciência do Solo vinculada a programas de pós-graduação na área específica apresenta-se incipiente na região amazônica, sendo necessárias ações de fomento e auxílio, com editais de demandas induzidas, que permitam o desenvolvimento dessa importante ciência. Nesse contexto, Ceretta, Anjos e Siqueira (2008) comentam que mesmo os grupos de pesquisas em Ciência do Solo são poucos na Região Norte, e isso sinaliza a necessidade da ampliação da área, especialmente por se tratar de ambientes de extrema importância e, em alguns casos, frágeis.

Quanto à expansão geográfica dos programas de pós-graduação de Ciência do Solo no Sul, Sudeste e Nordeste, de modo geral, eles acompanharam o desenvolvimento dos demais cursos que cresceram também nessas regiões, principalmente no Sudeste, a área de maior desenvolvimento econômico do País. Nesse contexto, Amaral (2011) disserta que o resultado não é diferente dos demais indicadores socioeconômicos, tais como o Índice de Desenvolvimento Humano (IDH), a renda per capita e o PIB social. É notável que a concentração de programas de pós-graduação em poucas regiões tenha demonstrando fortemente a regionalização das áreas de pesquisa, o que corrobora para que as diversidades intelectuais e os avanços científicos entre as regiões 
sejam mantidos, garantindo aos estados ricos mais conhecimentos e maior geração de riquezas e aos estados menos desenvolvidos a necessidade de importar tecnologias, o que tem ocorrido.

Além disso, o agrupamento dos programas de pós-graduação recomendados pela Capes e os desenvolvimentos econômicos das regiões indicam o potencial das pesquisas e da capacidade de transferência de tecnologia para a comunidade local. Esse fato impõe aos estados da região amazônica total ausência de acesso a programas de pós-graduação para estudos de questões relativas à Ciência do Solo com foco regional. Assim, para atender às necessidades e demandas locais, os empresários do agronegócio utilizam técnicas e conceitos produzidos em biomas diferentes, o que pode impor aos solos amazônicos condições desfavoráveis de manejo.

A situação atual da pós-graduação brasileira na região amazônica não difere da sua história. Desde o tempo do domínio português, foi introduzido ali um modelo fundamentado no extrativismo predatório, enquanto as bases para o desenvolvimento nacional foram implantadas principalmente nas regiões Sul e Sudeste do País. Contudo, a partir da década de 50 do século XX, algumas tentativas foram realizadas para tentar diminuir tal discrepância entre as regiões, como, por exemplo, o Plano de Valorização Econômica da Amazônia. Sem pessoal qualificado e sem recursos suficientes, essas tentativas não perseveraram, o que demonstra os primeiros sintomas da dificuldade do desenvolvimento de programas de pós-graduação na Amazônia, independentemente da área.

Apesar da expansão geográfica dos programas de pós-graduação em Ciência do Solo e do fato de eles não contemplarem a Região Norte do Brasil, foram observados 13 programas de pós-graduação com disciplinas relacionas à área (Figura 2 e Tabela 1), o que demonstra o reconhecimento da importância do tema para a região. Dos programas de pós-graduação estabelecidos na região e reconhecidos pela Capes, 53,8\% apresentam mestrado e doutorado e $46,5 \%$ apenas mestrado, sendo cinco cursos com conceito 3 , seis com conceito 4, dois com conceito 5 e nenhum com conceitos 6 e 7, considerando a última avaliação trienal 
(2010/2012). Isso demonstra que os programas de pós-graduação localizados na região amazônica estão em processo de consolidação.

Quando confrontados os resultados da conceituação desses programas com os dos demais programas de pós-graduação da região amazônica que não apresentam disciplinas da área de Ciência do Solo, verifica-se o mesmo comportamento, isto é, eles apresentam baixa conceituação em mais de 70\% dos cursos de mestrado (conceito 3 ) e apenas um programa de doutorado com conceito 6 , sendo a maioria (78\%) com conceito 4 . Tais resultados têm sido atribuídos à recente implantação de boa parte dos cursos.

Nota-se que há uma boa distribuição geográfica dos programas de pós-graduação com disciplinas relacionadas à Ciência do Solo, o que permite um desenvolvimento de conhecimento científico importante para a ocupação dos ecossistemas da região amazônica e reduz a assimetria do ensino de Ciência do Solo para a região. Apesar disso, há ainda uma inserção incipiente do tema Ciência do Solo nos programas existentes, reflexo do reconhecimento da comunidade científica local ligada ao tema para o desenvolvimento sustentável do Bioma Amazônico.

Portanto, a distribuição geográfica dos programas de pósgraduação da região é resultado das políticas públicas anteriores com foco na qualificação de técnicos especializados para o local. Observa-se que cinco cursos (38\%) pertencem ao Instituto Nacional de Pesquisas da Amazônia (Inpa), órgão criado na década de 50 em decorrência das preocupações com o estudo científico da região, incluindo a segurança nacional. O primeiro programa de pós-graduação data de 1971 e foi organizado pelo Inpa; em 1973, foi iniciada a pós-graduação na Universidade Federal do Pará (UFPA). 


\section{Tabela 1, Programas de Pós-Graduação (PPG) com disciplinas relacionadas com Ciência do Solo}

\begin{tabular}{|c|c|c|c|c|c|c|c|}
\hline $\mathbf{N}^{\circ}$ & PPG & Instituição & Estado & M & D & C. & URL \\
\hline 1 & $\begin{array}{l}\text { Ciência, } \\
\text { inovação e } \\
\text { tecnologia } \\
\text { para a } \\
\text { Amazônia }\end{array}$ & UFCA & $A C$ & $x$ & & 3 & http://www.ufac.br/portal \\
\hline 2 & $\begin{array}{l}\text { Produção } \\
\text { vegetal }\end{array}$ & UFCA & $A C$ & $x$ & & 3 & http://www.ufac.br/portal \\
\hline 3 & $\begin{array}{l}\text { Biologia } \\
\text { urbana }\end{array}$ & UniNilton & AM & $x$ & $x$ & 3 & http://www.niltonlins.br/ \\
\hline 4 & $\begin{array}{l}\text { Agricultura } \\
\text { no trópico } \\
\text { úmido }\end{array}$ & Inpa & AM & $x$ & & 3 & http://www.inpa.gov.br/ \\
\hline 5 & Entomologia & Inpa & AM & $x$ & $x$ & 5 & http://www.inpa.gov.br/ \\
\hline 6 & $\begin{array}{l}\text { Ciências de } \\
\text { florestas } \\
\text { tropicais }\end{array}$ & Inpa & AM & $x$ & $x$ & 5 & http://www.inpa.gov.br/ \\
\hline 7 & $\begin{array}{l}\text { Agronomia } \\
\text { tropical }\end{array}$ & Ufam & AM & $x$ & $x$ & 4 & http://www.ufam.edu.br/ \\
\hline 8 & Geociências & Ufam & AM & $x$ & & 3 & http://www.ufam.edu.br/ \\
\hline 9 & $\begin{array}{l}\text { Ciências } \\
\text { ambientais }\end{array}$ & UFPA & PA & $x$ & $x$ & 4 & http://www.portal.ufpa.br/ \\
\hline 10 & Agronomia & Ufra & PA & $x$ & $x$ & 4 & http://www.portal.ufra.edu.br/ \\
\hline 11 & Geografia & Unir & RO & $x$ & & 4 & http://www.unir.br/ \\
\hline 12 & Agronomia & UFRR & $\mathrm{RR}$ & $x$ & & 4 & http://ufrr.br/ \\
\hline 13 & $\begin{array}{l}\text { Produção } \\
\text { vegetal }\end{array}$ & UFT & TO & $x$ & $x$ & 4 & http:// www.uft.edu.br/ \\
\hline
\end{tabular}

Obs.: M: mestrado; D: doutorado; C: Conceito do Programa.

Em seguida, na década de 60, foi criada a Universidade Federal do Amazonas (Ufam), com o objetivo de capacitar pessoal para a consolidação de um novo cenário para a região. Essa universidade conta hoje com dois cursos que apresentam disciplinas relacionadas a solo, mas nenhum específico de Ciência do Solo.

Apesar das dificuldades, principalmente por falta de fixação de pessoal qualificado e pela sua rotatividade, nota-se, nessa distribuição de programas de pós-graduação, que se conseguiu introduzir mão de obra qualificada, professores e egressos dos programas, e, assim, contribuir 
para a capacitação de pessoal com conhecimento produzido localmente, o que é de extrema importância para o desenvolvimento sustentável regional.

A distribuição geográfica das instituições de ensino superior e, mais especificamente, de professores e alunos de pós-graduação pode integrar-se ao desenvolvimento sustentável por meio da geração de tecnologias apropriadas que favoreçam o uso dos recursos naturais locais, promovendo a inclusão social e a geração de renda, favorecendo a ampliação da soberania na região, sem perder o foco na sustentabilidade desses processos de uso dos recursos locais, algo que é realidade em muitas regiões do País e um mito em outras, por falta de políticas apropriadas.

Para uma análise mais profunda das disciplinas relacionadas à Ciência do Solo, foi necessário conhecer quais delas têm sido ministradas em cada instituição de ensino superior. Nesse sentido, constatou-se (Tabela 2) que as disciplinas ofertadas relacionadas à Ciência do Solo totalizam 39, mediadas por 36 docentes em 13 cursos. Esses dados norteiam a formação de um programa de pós-graduação em Ciência do Solo na região.

Tabela 2. Programas de pós-graduação, instituições e disciplinas ofertadas relacionadas à Ciência do Solo

Ciência, inovação e tecnologia para a Amazônia / UFCA: Ciência e tecnologia de argilominerais, micorrizas em solos tropicais, química e biogeoquímica de solos tropicais e solos amazônicos: gênese, natureza e utilidades.

Produção vegetal / UFCA: Biologia do solo, natureza e propriedades dos solos e nutrição mineral de plantas.

Biologia urbana / UniNilton: Ciclo de nutrientes e microrganismos do solo.

Agricultura no trópico úmido / Inpa: Biologia das terras pretas de índio da Amazônia central, fertilidade, nutrição de plantas na Amazônia, pedologia e solos da Amazônia.

Entomologia / Inpa: Fauna do solo.

Ciências de florestas tropicais / Inpa: Microbiologia do solo e ciclagem de nutrientes.

Agronomia tropical / Ufam: Nutrição de plantas e manejo e fertilidade de solos tropicais.

Geociências / Ufam: Planeta Terra.

Ciências ambientais / UFPA: Geoquímica do ambiente superficial, interação soloplanta-atmosfera, ciclo do carbono no contexto amazônico, mudanças de uso e cobertura da terra. 
Agronomia / Ufra: Nutrição mineral de plantas, química do solo, matéria orgânica, física do solo, manejo e conservação do solo e da água e gênese e morfologia, classificação do solo.

Geografia / Unir: Compactação de solos, uso e ocupação do solo na Pan-Amazônia e pedogeomorfologia.

Agronomia / UFRR: Microbiologia do solo, fertilidade do solo, física do solo, manejo e conservação do solo e água, pedologia e tópicos especiais na linha de pesquisa de manejo do solo e da água.

Produção vegetal / UFT: Manejo da fertilidade do solo e manejo e conservação do solo e da água.

Além da distribuição temática dos projetos em desenvolvimento nos programas de pós-graduação em Ciência do Solo e das disciplinas oferecidas, nota-se que há suporte teórico para atender as cinco temáticas abordadas em âmbito nacional. Ressalte-se que $26 \%$ das disciplinas oferecidas referem-se à fertilidade do solo/adubação e nutrição, 23\% ao manejo e à conservação do solo e da água, 20\% à biologia e bioquímica, $26 \%$ à pedologia e $5 \%$ a recursos naturais e qualidade ambiental e reciclagem.

Com relação às características do perfil dos profissionais, professores/pesquisadores (36) dos diferentes programas de pósgraduação que lecionam disciplinas relacionadas à Ciência do Solo, o primeiro componente a ser considerado é referente à formação universitária. Nesse contexto, considerando todos os programas de pósgraduação avaliados (17), vê-se que $47 \%$ dos professores/pesquisadores obtiveram seu título de mestre em Ciência do Solo e $13(36 \%)$ doutores (Tabela 3), demonstrando forte aderência à temática, além de evidenciar potencial para a criação de um programa de pós-graduação em Ciência do Solo multi-institucional.

Quanto à formação recebida pelos docentes, constatou-se que $36 \%$ dos títulos de mestre e doutor foram obtidos na Universidade Federal de Viçosa e na Universidade de São Paulo. Tais dados chamam atenção, pois a maioria desses docentes foram egressos de cursos com alta conceituação da Capes. Esse é mais um indicativo da qualidade do profissional da região, além de apontar a possibilidade de parcerias com instituições renomadas do Brasil no que tange não só a pesquisas, mas também a intercâmbio para a qualificação da mão de obra regional. 
Quanto às demais formações recebidas por outros docentes atuantes também nas disciplinas de Ciência do Solo, e tendo as diversas linhas de pesquisa dessa área do conhecimento para contextualizálas nos aspectos ambientais, científicos e socioculturais, elas são indispensáveis para uma formação multidisciplinar e acadêmica. Nota-se (Tabela 3) que a formação desses docentes/pesquisadores tem contribuído para alavancar pesquisas relevantes para o Bioma Amazônico, na área de Ciência do Solo.

Tabela 3. Porcentagem de profissionais em função da titulação

\begin{tabular}{|c|c|c|c|}
\hline \multirow{2}{*}{ Cursos } & Graduação & Mestrado & Doutorado \\
\hline & \multicolumn{3}{|c|}{$\%$} \\
\hline Agronomia & 67 & 11 & 11 \\
\hline Biotecnologia & & 3 & 6 \\
\hline Botânica & & & 3 \\
\hline Ciência agrária & & & 3 \\
\hline Ciência agroquímica & & & 3 \\
\hline Ciência atmosférica & & 3 & 3 \\
\hline Ciência biológica & 5 & 8 & \\
\hline Ciência do solo & & 44 & 31 \\
\hline Energia elétrica & & & 3 \\
\hline Ciência florestal & & 6 & \\
\hline Energia nuclear na agricultura & & & 3 \\
\hline Entomologia & & & 3 \\
\hline Fitotecnia & 8 & 6 & 8 \\
\hline Geociência & & & 3 \\
\hline Geografia & & & 3 \\
\hline Geodinâmica & & 3 & \\
\hline Geologia & 10 & 6 & 6 \\
\hline Matemática & 5 & 3 & \\
\hline Metereologia & 5 & 6 & 6 \\
\hline Sensoriamento remoto & & 3 & \\
\hline Microbiologia agrícola & & & 6 \\
\hline Tecnologia energética nuclear & & & 3 \\
\hline
\end{tabular}

Assim como se espera que a formação acadêmica possua ciência, teoria, prática e postura ética, acredita-se que o ambiente funcional dos docentes/pesquisadores suporta a criação de um programa de pós- 
graduação dentro do que estabelecem os padrões de exigência da Capes. Nesse sentido, Berbel (1998) propõe como premissa a necessidade de se dotar o aluno de sensibilidade para detectar problemas, a fim de que, então, possa assumir postura mais consciente em cada caso. Premissa importante quando se pretende trabalhar em um ambiente vulnerável, com elevada intervenção antrópica, potencialmente frágil e, principalmente, de importância político-social internacional.

\section{Considerações finais}

Tendo em vista o número de docentes atuantes e sua formação acadêmica, algumas questões são pertinentes: existe a possibilidade de criação de um programa de pós-graduação em Ciência do Solo na região amazônica? Ou de criação de um programa interinstitucional em Ciência do Solo?

Obviamente, são inúmeras as dificuldades de se obter respostas para tais questionamentos, sobretudo por se tratar de uma área de estudo complexa e que exige dos docentes grandes contribuições, pois conduzir um programa de pós-graduação em Ciência do Solo é uma tarefa árdua.

Certamente, reconhece-se que, apesar do maior número dos docentes serem mestres e doutores em Ciência do Solo ou formados em universidades com bons conceitos de acordo com a Capes, como, por exemplo, a Universidade Federal de Viçosa e a Universidade de São Paulo, quando confrontadas as disciplinas e as temáticas trabalhadas em âmbito nacional, observa-se que apenas 5\% trabalham com qualidade do solo e reciclagem. Aí está o primeiro desafio, uma vez que a Região Norte é um ambiente importante e, em alguns casos, frágil, onde têm avançado as fronteiras agrícolas.

A introdução dessa área de conhecimento pode contribuir de forma mais rápida e com qualidade para o desenvolvimento sustentável da Região Norte do Brasil, especialmente da Amazônia. Sobretudo porque ali se encontra a Floresta Amazônica, ambiente natural diversificado, em especial quanto à fauna e flora, o que o torna estimulante para as 
pesquisas. Essa iniciativa poderá ainda favorecer a integração dessa região aos processos de desenvolvimento sustentável, isto é, apropriarse de tecnologia e inovações para o uso de produtos da floresta com vistas à inclusão social e à geração de renda, além de qualificar pessoal em nivel de pós-graduação para os povos amazônicos.

Espera-se, portanto, que este estudo possa contribuir para o avanço da discussão sobre o cenário atual da inserção de programa de pós-graduação stricto sensuem Ciência do Solo na Região Norte, uma vez que ficou constatado que nessa região se encontram profissionais com habilidade e competências para realizar tal tarefa, boas universidades, institutos de pesquisas competentes e um Núcleo Regional da Sociedade Brasileira de Ciência do Solo atuante. Nesse sentido, destacam-se políticas com ações voltadas para este fim, como:

a) Fortalecimento dos grupos de pesquisa em Ciência do Solo e intercâmbio entre eles, formando uma rede de pesquisa na região amazônica, com foco em desenvolvimento da Ciência do Solo regional;

b) Incentivo e financiamento para a vinda de pesquisadores de competência comprovada em Ciência do Solo, nacional e/ou internacionalmente, na condição de visitante;

c) Fomento às pesquisas, com editais específicos, com foco regional para essa região, como já ocorre;

d) Criação e/ou fortalecimento de laboratórios de análises com foco na temática Ciência do Solo, multiusuários e multi-institucionais;

e) Abertura e desenvolvimento inicial de um programa de pósgraduação em Ciência do Solo (Proap e bolsas de estudo específicas para essa região) com apoio da Capes; e

f) Promoção de parcerias com programas de pós-graduação em Ciência do Solo consolidados (Procad/Capes e Casadinho/CNPq/Capes).

Essas ações contribuirão para a redução das assimetrias, geração de pesquisas de importância regional, formação de recursos humanos de elevada qualidade e fixação de doutores na região.

As proposições desta pesquisa se tornam ainda mais prementes quando se avaliam as distâncias entre os polos de pesquisa, bem como as dificuldades físicas de acesso a esses polos, o que exigirá 
ações governamentais diferentes daquelas estabelecidas nos centros tradicionais de pesquisa.

Recebido em 31/05/2013

Aprovado em: 17/03/2014

\section{Referências bibliográficas}

ACHARD, F. D. et al. Determination of deforestation rates of the world's humid tropical forests. Science, v. 297, n. 5583, p. 999-1002, 2002.

AMARAL, E. F. L. Notas de aula - Principais conceitos de indicadores sociais. 2011. Disponivel em: <http://www.ernestoamaral.com/docs / dcp033-111/Aulas17-19.pdf>. Acesso em: 20 jan. 2013.

BERBEL, N. N. Problematization and Problem-Based Learning: different words or different ways? Interface - Comunicação, Saúde, Educação, v. 2, n. 2, 1998.

CARNEIRO, M. A. C.; SOUZA, E. D.; PAULINO, H. B. Pós-graduação em Ciência do Solo no Brasil. Boletim Informativo SBCS, v. 36, n. 2, 2011.

CERETTA, C. A.; ANJOS, L. H. C.; SIQUEIRA, J. O. A pós-graduação em Ciência do Solo no Brasil: evolução e tendências. RBPG, Brasília, v. 5, n. 9, p. 7-35, dez. 2008.

CAPES - Coordenação de Aperfeiçoamento de Pessoal de Nivel Superior. Cursos recomendados e reconhecidos, 2009-2012. Disponível em: <http://www.capes.gov.br/cursos-recomendados>. Acesso em: 10 jan. 2013.

FAO - Organização das Nações Unidas para a Alimentação e Agricultura. Plantio direto no Sul do Brasil. 2012. Relatório. Disponível em: <www. fao.org.br>. Acesso em: 01 abr. 2014.

Relatório. 2013. Disponível em: <www.fao.org.br>. Acesso em: 15 fev. 2013. 
LAURANCE, W. F. et al. The Future of the Brazilian Amazon. Science, v. 291, n. 5503, p. 438-439, 2001.

MITTERMEIER, R. A. et al. Conservation of primates in the Atlantic Forests of Brazil. Int. Zoo. Yearbook, v. 22, p. 2-17, 1982.

MORAES, M. H. M.; GIROLDO, D. Distribuição geográfica da pós-graduação: estudo de Indicadores. Seminário de Pesquisa em Educação da Região Sul, 2012.

MANZATTO, C. V.; FREITAS JUNIOR, E. ; PERES, J. R. R (Ed.). Uso agrícola dos solos brasileiros. Rio de Janeiro: Embrapa Solos, 2002.

OLIVER, R. W.; DANIEL, C. R.; ROBERT, M. E. Extinction debt and windows of conservation opportunity in the Brazilian Amazon. Science, v. 337, n. $13,2012$. 